\title{
Using a clicker question sequence to teach time-development in quantum mechanics
}

\author{
Peter $\mathrm{Hu}$, Yangqiuting Li, and Chandralekha Singh \\ Department of Physics and Astronomy, University of Pittsburgh, Pittsburgh, PA 15260
}

Research-validated clicker questions as instructional tools for formative assessment are relatively easy to implement and can provide effective scaffolding when developed and implemented in a sequence. We present findings from the implementation of a research-validated Clicker Question Sequence (CQS) on student understanding of the time-development of two-state quantum systems. This study was conducted in an advanced undergraduate quantum mechanics course. The effectiveness of the CQS was determined by evaluating students' performance after traditional lecture-based instruction and comparing it to their performance after engaging with the CQS.

2021 PERC Proceedings edited by Bennett, Frank, and Vieyra; Peer-reviewed, doi.org/10.1119/perc.2021.pr.Hu Published by the American Association of Physics Teachers under a Creative Commons Attribution 4.0 license. Further distribution must maintain the cover page and attribution to the article's authors. 


\section{INTRODUCTION}

The time-evolution of a quantum state is an important concept in quantum mechanics. Many fields of active research, including quantum computing, must contend with the dynamical behavior of quantum systems. Since it draws on prerequisite knowledge of quantum states and the Hamiltonian of the system, the concept can be challenging for students to grasp. At the advanced undergraduate level, time-evolution of a quantum state is introduced with a timeindependent Hamiltonian $\widehat{H}$. The state as a function of time $t$ is then the solution to the time-dependent Schrödinger equation, i.e., $i \hbar \frac{d}{d t}|\Psi(t)\rangle=\widehat{H}|\Psi(t)\rangle$, and is equivalent to applying the operator $e^{-\frac{i \widehat{H} t}{\hbar}}$ to the initial state.

Because the Hamiltonian governs the time-development of the state, the eigenstates of the Hamiltonian, i.e., the energy eigenstates or "stationary states," are special in that they simply acquire an overall time-dependent phase factor. For instance, given a Hamiltonian $\widehat{H}=C \hat{S}_{z}$ for a two-state system with a dimensionally-appropriate constant $C$, an initial state expressed in the energy eigenbasis is $|\chi(t=0)\rangle=a|z\rangle+b|-z\rangle$. The state at time $t$ is $|\chi(t)\rangle=$ $a e^{-\frac{i C t}{2}}|z\rangle+b e^{\frac{i C t}{2}}|-z\rangle$, since $\widehat{H}|z\rangle=\frac{C \hbar}{2}|z\rangle$ and $\widehat{H}|-z\rangle=$ $-\frac{C \hbar}{2}|-z\rangle$. Here all notations are standard. If, however, the initial state is expressed in some other basis, one can obtain the state at time $t$ by first re-expressing the initial state as a superposition of energy eigenstates before introducing the time-dependent phase factors to each term.

To become proficient at determining the state at time $t$ given an initial state in some basis, students must be adept at several different tasks. These include being able to recognize whether the given initial state is an eigenstate of the Hamiltonian; working in the energy eigenbasis and converting to this basis if the initial state is given in any other basis; and correctly applying the time-evolution operator. Students also must recognize that different energy eigenstates generally correspond to different eigenvalues. The convergence of all these challenges, as well as possible unfamiliarity with the meaning of the complex exponential itself, can place significant demands on students' cognitive resources. This may also obfuscate other consequences of the Hamiltonian playing a central role, e.g., the expectation value of any observable (that does not have any explicit timedependence) does not depend on time in a stationary state.

Prior research suggests that students in quantum mechanics courses often struggle with many common difficulties, including with issues related to timedevelopment of a quantum state, but research-validated learning tools can effectively help students develop a robust knowledge structure [1-37]. Quantum Interactive Learning Tutorials (QuILTs) have been developed, validated and implemented on many topics in quantum mechanics, with encouraging results $[19,38,39]$. Similarly, clicker questions, first popularized by Mazur using his Peer Instruction method, are conceptual multiple-choice questions presented to a class for students to answer anonymously, individually first and then again after discussion with peers, and with immediate feedback. They have proven effective and are relatively easy to incorporate into a typical course, without the need to greatly restructure classroom activity or assignments [40,41]. When presented in sequences of validated questions that build on one another, they can systematically help students with a particular theme that they may be struggling with. Previously, such Clicker Question Sequences (CQS) have been developed, validated and implemented on several key topics in quantum mechanics [42-46]. Here we discuss the development, validation and implementation of a CQS focused on helping students learn time-evolution of two-state quantum systems.

\section{METHODOLOGY}

The CQS targets upper-level students in junior-/seniorlevel quantum mechanics courses. The data presented here is from implementation in a mandatory junior-/senior-level course at a large research university, with sample size $\mathrm{N}=$ 29. To develop and validate this CQS, we took advantage of the learning objectives and goals of the QuILT on this topic that had previously been developed $[38,39,45]$. Taking inspiration from the validated pre- and post-tests intended for use with that QuILT, we made adjustments to questions to specifically address the time-development of two-state systems. Additional inspiration came from questions from other sequences, including those focused on the timedevelopment in the context of Larmor precession.

Additionally, we took advantage of much of the cognitive task analysis both from the expert and student perspectives (based upon interviews) and the scaffolding that had been incorporated in the aforementioned QuILT. We focused on condensing this material, to ensure that the CQS can be administered in class. To be strategic with regard to the available class time, we prioritized basic conceptual knowledge and specific consequences that students often find difficult, provided checkpoints at which instructors should discuss some broader themes related to the previous questions, and avoided burdensome calculations.

After we conceptualized the most important features of time-development of quantum states that students should know, we drafted questions and discussed among ourselves many times to minimize unintended interpretations. We standardized terminologies and sentence constructions while simplifying them as much as possible to avoid causing cognitive overload for students. We also paid attention to the answer choices for each question. In some instances, after discussion amongst researchers, we revised the questions to make sure that students understood them unambiguously.

We aimed to address common stumbling blocks and emphasized key features that students may have missed in a typical lecture. The 13 questions in the CQS focused on four learning goals on the following topics: identifying the basic 
properties of the energy eigenstates or stationary states (CQS 1.X, 2 questions), transforming from an initial state to its time-evolved state (CQS 2.X, 5 questions), expressing a state in the energy eigenbasis before applying the time-evolution operator (CQS 3.X, 4 questions), and calculating the timedependence of various observables' expectation values (CQS 4.X, 2 questions). We designed several questions specifically to address certain student difficulties that have previously been found $[12,13,20]$. Selected CQS questions, referenced in later sections, are reproduced below (answers in boldface, all notations being standard and familiar to students):

CQS 1.2Consider a system with a Hamiltonian $\widehat{H}=C \hat{S}_{z}$. Which of the following initial states $|\chi(t=0)\rangle$ is a stationary state?

$$
\begin{aligned}
\text { I. } & & |\chi(0)\rangle & =|z\rangle \\
\text { II. } & & |\chi(0)\rangle & =|x\rangle
\end{aligned}
$$

III. $\quad|\chi(0)\rangle=a|z\rangle+b|-z\rangle$, because $|z\rangle$ and $|-z\rangle$ are both stationary states.
A. All of the above
B. I only
C. I and II only
D. I and III only
E. None of the above

CQS 2.2Consider a system with a Hamiltonian $\widehat{H}=C \hat{S}_{z}$. Choose all the correct statements about a system in the state $|\chi(0)\rangle$.

I. $|\chi(t)\rangle=e^{\frac{-i E_{n} t}{\hbar}}|\chi(0)\rangle$, where $E_{n}$ is an eigenvalue of $\widehat{\mathrm{H}}$.

II. $|\chi(t)\rangle=e^{\frac{-i \hat{H} t}{\hbar}}|\chi(0)\rangle$, where $\widehat{H}$ is the

Hamiltonian of the system.

III. Each measurement of a generic observable $Q$ will return the same result, regardless of the time when the measurement is performed.
A. II only
B. III only
C. I and II only
D. II and III only
E. None of the above

CQS 3.1Consider a system with a Hamiltonian $\widehat{H}=C \hat{S}_{z}$. Choose all the correct statements about a system in the state $|\chi(0)\rangle$.

I. $|\chi(0)\rangle=(|z\rangle\langle z|+|-z\rangle\langle-z|)|\chi(0)\rangle$, where $|z\rangle\langle z|+|-z\rangle\langle-z|=\hat{I}$

II. $|\chi(0)\rangle=C_{1}|z\rangle+C_{2}|-z\rangle$, where $C_{1}=\langle z \mid \chi(0)\rangle$

and $\quad C_{2}=\langle-z \mid \chi(0)\rangle$ are both coefficients

III. $|\chi(t)\rangle=e^{\frac{-i E_{+} t}{\hbar}} C_{1}|+z\rangle+e^{\frac{-i E_{-} t}{\hbar}} C_{2}|-z\rangle$
A. I only
B. II only
C. I and II only
D. II and III only
E. All of the above

CQS 3.2Consider a system with a Hamiltonian $\widehat{H}=C \hat{S}_{z}$. Choose all of the following that are correct about a system in the state $|\chi(0)\rangle=a|x\rangle+b|-x\rangle$.

$\begin{aligned} & \text { I. } & |\chi(t)\rangle & =e^{\frac{-i \widehat{H} t}{\hbar}}|\chi(0)\rangle \\ & \text { II. } & |\chi(t)\rangle & =a e^{\frac{-i E_{+} t}{\hbar}}|x\rangle+b e^{\frac{-i E_{-} t}{\hbar}}|-x\rangle\end{aligned}$

III. To find $|\chi(t)\rangle$, we can write $|\chi(0)\rangle$ as a linear superposition of energy eigenstates, and then attach a time-dependent phase factor with the appropriate energy to each term.
A. I only
B. III only
C. I and II only
D. I and III only
E. All of the above

CQS 3.3Consider a system with a Hamiltonian $\widehat{H}=C \hat{S}_{z}$. Choose the correct expression for the time evolved state $|\chi(t)\rangle$ given an initial state $|\chi(0)\rangle=a|x\rangle+b|-x\rangle$.
A. $|\chi(t)\rangle=a e^{\frac{-i E_{+} t}{\hbar}}|x\rangle+b e^{\frac{-i E_{-} t}{\hbar}}|-x\rangle$
B. $|\chi(t)\rangle=\frac{a}{\sqrt{2}} e^{\frac{-i E_{+} t}{\hbar}}|z\rangle+\frac{b}{\sqrt{2}} e^{\frac{-i E_{-} t}{\hbar}}|-\mathrm{z}\rangle$
C. $|\chi(t)\rangle=\frac{a+b}{\sqrt{2}} e^{\frac{-i E_{+} t}{\hbar}}|x\rangle+\frac{a-b}{\sqrt{2}} e^{\frac{-i E_{-} t}{\hbar}}|-x\rangle$
D. $|\chi(t)\rangle=\frac{a+b}{\sqrt{2}} e^{\frac{-i E_{+} t}{\hbar}}|z\rangle+\frac{a-b}{\sqrt{2}} e^{\frac{-i E_{-} t}{\hbar}}|-z\rangle$
E. None of the above

CQS 3.4Consider a system with a Hamiltonian $\widehat{H}=C \hat{S}_{x}$. Choose all of the following that are correct about the time development of the state $|\chi(0)\rangle=\frac{1}{\sqrt{2}}|z\rangle+\frac{1}{\sqrt{2}}|-z\rangle$.

I. $|\chi(t)\rangle=\frac{1}{\sqrt{2}} e^{\frac{-i E_{+} t}{\hbar}}|z\rangle+\frac{1}{\sqrt{2}} e^{\frac{-i E_{-} t}{\hbar}}|-z\rangle$

II. $|\chi(t)\rangle=\frac{1}{\sqrt{2}} e^{\frac{-i E_{+} t}{\hbar}}|x\rangle+\frac{1}{\sqrt{2}} e^{\frac{-i E_{-} t}{\hbar}}|-x\rangle$

III. $|\chi(t)\rangle=e^{\frac{-i E_{+} t}{\hbar}}|x\rangle$
A. I only
B. II only
C. III only
D. I and III only
E. None of the above

CQS 4.2Consider a system with a Hamiltonian $\widehat{H}=C \hat{S}_{z}$. If the system is in the state

$$
|\chi(0)\rangle=|z\rangle,
$$

which of the following expectation values are time-independent?
I. Energy II. $S_{z}$
A. None of the above
C. I and II only
III. $S_{x}$
B. I only
D. II and III only

E. All of the above

Since the entire course was remote due to the COVID-19 pandemic, the CQS was administered during the online lectures as a Zoom poll while the instructor displayed the questions via the "Share Screen" function. The instructor allowed several minutes for students to vote before revealing the results, and some had the opportunity to explain their responses, before systematically discussing the different options. When a majority of students selected an option that involved alternative conceptions, the instructor would give a hint and ask students to vote again, and ask for volunteers to explain the reasoning behind their choices. In a typical classroom setting, students would have had easy access to one another to discuss their thinking in small groups, but this proved less feasible in the online instructional setting, where students or the instructor predominantly spoke to the whole class.

To determine the effectiveness of the CQS in helping students overcome these common difficulties, we developed and validated pre- and post-tests that had both questions 
taken directly from the CQS and other questions on topics covered in the CQS. The post-tests were a slightly modified version of the pre-tests, with some changes (e.g., eigenstates of $\hat{S}_{x}$ being replaced by eigenstates of $\hat{S}_{y}$ ) but otherwise remaining conceptually similar. Students were given the pretest immediately following traditional lecture-based instruction on the topic. After administration of the CQS, which took place over the course of three lecture sessions, students were given the post-test. For both, they were given a 25-minute period at the end of the class session. Two researchers graded the pre-test and post-test, and after discussion converged on a rubric on which the inter-rater reliability was greater than 95\%. Questions 3 and 4 were scored with 2 points split between answer and reasoning, and the remainder were all-or-nothing. The pre- and post-test questions are reproduced below:

Q1. (See CQS 2.2)

Q2. Consider a system with a Hamiltonian $\widehat{H}=C \hat{S}_{z}$, where $C$ is an appropriate constant. Choose all of the following that are stationary states.

$$
\begin{array}{ll}
\text { I. } & \frac{1}{\sqrt{2}}|z\rangle+\frac{1}{\sqrt{2}}|-z\rangle \\
\text { II. } & a|z\rangle+b|-z\rangle \\
\text { III. } & a|x\rangle+b|-x\rangle, \text { where } a \neq b
\end{array}
$$
a. All of the above
b. I only
c. II only
d. I and II only
e. None of the above

Q3. Consider a system with a Hamiltonian $\widehat{H}=C \hat{S}_{x}$ (note: $\underline{n o t} \widehat{H}=C \hat{S}_{z}$ ), where $C$ is an appropriate constant. For a system in the state $|\chi(0)\rangle=\frac{1}{\sqrt{5}}|x\rangle+\frac{2}{\sqrt{5}}|-x\rangle$, what is $|\chi(t)\rangle$ ? Show your work.

Q4. Consider a system with a Hamiltonian $\widehat{H}=C \hat{S}_{x}$, where $C$ is an appropriate constant. For a system in the state $|\chi(0)\rangle=\frac{1}{\sqrt{5}}|z\rangle+\frac{2}{\sqrt{5}}|-z\rangle$, what is $|\chi(t)\rangle$ ? Show your work.

Q5. Consider a system with a Hamiltonian $\widehat{H}=C \hat{S}_{z}$, where $C$ is an appropriate constant.

For a system in the state $a|z\rangle+b|-z\rangle$, which of the following expectation values are time-independent?
I. Energy
II. $S_{y}$
III. $S_{z}$
a. None of the above
b. I only
c. I and III only
d. II and III only
e. All of the above

Q6. Consider a system with a Hamiltonian $\widehat{H}=C \hat{S}_{z}$, where $C$ is an appropriate constant.

For a system in the state $|z\rangle$, which of the following expectation values are time-independent?
I. Energy
II. $S_{y}$
a. None of the above
III. $S_{z}$
c. I and III only
e. All of the above
b. I only
d. II and III only

\section{IN-CLASS IMPLEMENTATION RESULTS}

In addition to examining the improvement from the pretest to the post-test, we analyzed student performance on the clicker questions, including the attractiveness of the distractors. The pre-test and post-test results, as well as normalized gain [2] and effect sizes [47], are listed in Table I. The effect sizes for the six questions ranged from 0.45 to over 1 , indicating conventionally medium to large effects.

TABLE I. Comparison of pre- and post-test scores, along with normalized gain $(\mathrm{g})$ and effect size as measured by Cohen's $d$ (d), for students who engaged with the CQS $(\mathrm{N}=29)$.

\begin{tabular}{ccccc}
\hline \hline Q\# & $\begin{array}{c}\text { Pre-test } \\
\boldsymbol{\mu}\end{array}$ & $\begin{array}{c}\text { Post-test } \\
\boldsymbol{\mu}\end{array}$ & $\mathbf{g}$ & $\mathbf{d}$ \\
\hline Q1 & $28 \%$ & $72 \%$ & 0.62 & 0.99 \\
Q2 & $24 \%$ & $72 \%$ & 0.64 & 1.08 \\
Q3 & $67 \%$ & $83 \%$ & 0.47 & 0.45 \\
Q4 & $40 \%$ & $71 \%$ & 0.51 & 0.72 \\
Q5 & $24 \%$ & $69 \%$ & 0.59 & 0.99 \\
Q6 & $24 \%$ & $59 \%$ & 0.45 & 0.73 \\
\hline \hline
\end{tabular}

Many of the common student difficulties were successfully addressed to varying degrees after students engaged with CQS, as follows.

\section{A. Difficulties that decreased significantly after CQS}

\section{Difficulties with general eigenstates vs. stationary states}

The highest normalized gains were seen in the questions that probed students' knowledge of stationary states $(\mathrm{Q} 1$ and Q2). The energy eigenstates are stationary states, but students often remember "eigenstates" as stationary states without having recognized the importance of the "energy eigenstates" aspect. These difficulties could be exacerbated if students are shaky on the prerequisite linear algebra, without a clear grasp of what eigenstates and operators mathematically or conceptually are. Moreover, even if students are proficient with the linear algebra in the context of a math course, transferring that knowledge to the context of a quantum mechanics course can still be very challenging. As illustrated by CQS question 2.2, which also appeared as Q1 on the pre-test and post-test, students appeared to understand the distinction between generic eigenstates and energy eigenstates after the CQS. As seen in Table I, $28 \%$ of students answered this question correctly on the pre-test; $40 \%$ answered correctly during the CQS (not shown); and $72 \%$ answered correctly on the post-test, indicating substantial improvement. Additionally, on Q2, students also better recognized that a superposition of stationary states is not a stationary state, with a normalized gain of 0.64 . With similar normalized gains and effect sizes, students also learned in Q5 that only the expectation value of energy does not vary with time in a non-stationary state. 


\section{Replacing the operator $\widehat{H}$ with one eigenvalue $E_{n}$}

For CQS questions 2.2-2.5, students chose with substantial frequency an answer option that resulted in a single phase term involving energy instead of a sum of terms (distractor choice I in question 2.2 invokes this idea). This is at least partially due to students not being entirely comfortable with the notation or not understanding the role of the Hamiltonian. After CQS instruction, most students, when asked in the free-response Q3 on the post-test, correctly multiplied each energy eigenstate by a separate phase factor, with a normalized gain of 0.47 (see Table I).

\section{Difficulties with change of basis}

The CQS question 3.4, which asked students to change from the $z$-basis to the $x$-basis, had considerably lower performance than the preceding question 3.3, which had asked the reverse. This is at least partly due to students having less experience with the former transformation, as the latter is the predominant example used to introduce the idea of changing basis. The symmetry between the two cases may be obvious to more experienced problem solvers, but students needed the opportunity to reason through the basis change. Once students learned the importance of working in the appropriate basis, addressed in CQS questions 3.1-3.4, more students correctly answered the corresponding question $(\mathrm{Q} 4)$ on the post-test, with normalized gain 0.51 . We note that the performance on $\mathrm{Q} 4$, with an average of $71 \%$, is a bit lower than that on Q3, 83\%, as shown in Table I. With the exception of the latter expressing the given state in the correct basis, the two questions were identical. The lower performance on Q4 is likely due to forgetting the basis change, or making a mistake in the process.

\section{B. Difficulties that were less successfully addressed}

Improvement on Q6 on the post-test was the weakest, as seen in Table I. The question appeared with small changes on the pre-test and post-test, and as CQS question 4.2. Although there was some improvement from the pre-test to the CQS question, evidence from the post-test suggests very little further improvement. Students must recognize that invariant probabilities of measurement outcomes imply static expectation values, though it appears that more scaffolding is needed to help students learn this concept. Moving forward, we would specifically include suggestions to encourage students to think of an expectation value as an average of a large number of measurements made on identically prepared systems. Students could also benefit from an additional discussion of Ehrenfest's theorem, giving them more tools with which to process these ideas [29].

\section{Several examples of class discussion}

A particular advantage of the CQS is that it provides opportunity for rich class discussions that can deepen student understanding. Following are examples of such discussion.
Question 1.2 addressed the common difficulty that any superposition of stationary states is itself a stationary state. Initially the correct answer was not even the most popular response. Without immediately giving a full explanation, the instructor noted that, since any state can be written as a superposition of stationary states, selecting this option would imply that every possible state is a stationary state. When the class was allowed to vote a second time, nearly $50 \%$ chose the correct answer.

On question 3.2, two students volunteered to explain how the time evolution of the state could not be simplified (expressed without the Hamiltonian operator) by remaining in the $\{|x\rangle,|-x\rangle\}$ basis, and could thus rule out option II.

Question 4.2 asks about the time-dependence of the expectation value of an observable in a stationary state. Despite the instructor's hints, the distribution of answers remained nearly identical both times the polling was opened to students. While the students may not have been able to sufficiently parse the hints individually, it is likely that performance would have improved in a typical classroom setting, if students were given an opportunity to discuss the meaning of the hints and their consequences in small groups.

Opportunities to hold an overall class discussion about salient concepts such as these after students have voted are very important, but ensuring that instructors hold such discussions when they are recommended can be a challenge especially because time is limited. We will continue to investigate ways to encourage such discussions via check points between CQS questions, even in instances when the instructor may opt not to follow our suggestions verbatim.

\section{SUMMARY}

Clicker question sequences can be effective when implemented alongside traditional classroom lectures. We developed, validated, and found encouraging results from implementation of a CQS on the topic of time-development in two-state systems. Post-test scores improved for every question following the administration of the CQS, with mostly uniform normalized gains of around 0.60 in the multiple-choice questions, and high performance in the generative questions that asked students to correctly apply the time-development concepts in open-ended questions. Effect sizes throughout are conventionally large to medium: most were over 0.70. Students' performance was weakest on the questions on expectation values, but we believe that this can be improved through a more robust classroom discussion and more focus on this topic in the CQS itself.

We emphasize that this study was conducted in a remote learning context, and that these results may not transfer exactly to traditional classroom instruction contexts. We will investigate this further in the future.

\section{ACKNOWLEDGEMENTS}

We thank the NSF for award PHY-1806691. 
[1] D. F. Styer, Common misconceptions regarding quantum mechanics, Am. J. Phys. 64, 31 (1996).

[2] R. Hake, Interactive-engagement versus traditional methods: A six-thousand-student survey of mechanics test data for introductory physics courses, Am. J. Phys. 66, 64 (1998).

[3] P. Jolly, D. Zollman, S. Rebello, and A. Dimitrova, Visualizing potential energy diagrams, Am. J. Phys. 66, 57 (1998).

[4] I. Johnston, K. Crawford, and P. Fletcher, Student difficulties in learning quantum mechanics, Int. J. Sci. Educ. 20, 427 (1998).

[5] C. Singh, Student understanding of quantum mechanics, Am. J. Phys. 69, 885 (2001).

[6] R. Müller and H. Wiesner, Teaching quantum mechanics on an introductory level, Am. J. Phys. 70, 200 (2002).

[7] M. Wittmann, R. Steinberg, and E. Redish, Investigating student understanding of quantum physics: Spontaneous models of conductivity, Am. J. Phys. 70, 218 (2002).

[8] D. A. Zollman, N. S. Rebello, and K. Hogg, Quantum mechanics for everyone: Hands-on activities integrated with technology, Am. J. Phys. 70, 252 (2002).

[9] D. Domert, C. Linder, and Å. Ingerman, Probability as a conceptual hurdle to understanding one-dimensional quantum scattering and tunnelling, Eur. J. Phys. 26, 47 (2004).

[10] C. Singh, Transfer of Learning in Quantum Mechanics, AIP Conference Proceedings 790, 23 (2005).

[11] C. Singh, M. Belloni, and W. Christian, Improving students' understanding of quantum mechanics, Phys. Today 59, 43 (2006).

[12] C. Singh, Student Difficulties with Quantum Mechanics Formalism, AIP Conference Proceedings 883, 185 (2007).

[13] C. Singh, Student understanding of quantum mechanics at the beginning of graduate instruction, Am. J. Phys. 76, 277 (2008).

[14] C. Singh and G. Zhu, Cognitive Issues in Learning Advanced Physics: An Example from Quantum Mechanics, AIP Conference Proceedings 1179, 63 (2009).

[15] A. Kohnle, M. Douglass, T. J. Edwards, A. D. Gillies, C. A. Hooley, and B. D. Sinclair, Developing and evaluating animations for teaching quantum mechanics concepts, Eur. J. Phys. 31, 1441 (2010).

[16] G. Zhu and C. Singh, Surveying students' understanding of quantum mechanics in one spatial dimension, Am. J. Phys. 80, 252 (2012).

[17] G. Zhu and C. Singh, Improving students' understanding of quantum measurement. I. Investigation of difficulties, Phys. Rev. ST Phys. Educ. Res. 8, 010117 (2012).

[18] G. Zhu and C. Singh, Improving students' understanding of quantum measurement. II. Development of research-based learning tools, Phys. Rev. ST Phys. Educ. Res. 8, 010118 (2012).

[19] G. Zhu and C. Singh, Improving student understanding of addition of angular momentum in quantum mechanics, Phys. Rev. ST Phys. Educ. Res. 9, 010101 (2013).

[20] E. Marshman and C. Singh, Investigating Student Difficulties with Time dependence of Expectation Values in Quantum Mechanics, PERC Proc. (2013), pp. 245-248, https://doi.org/10.1119/perc.2013.pr.049.
[21] A. Kohnle, I. Bozhinova, D. Browne, M. Everitt, A. Fomins, P. Kok, G. Kulaitis, M. Prokopas, D. Raine, and E. Swinbank, A new introductory quantum mechanics curriculum, Eur. J. Phys. 35, 015001 (2013).

[22] P. J. Emigh, G. Passante, and P. S. Shaffer, Student understanding of time dependence in quantum mechanics, Phys. Rev. ST Phys. Educ. Res. 11, 020112 (2015).

[23] E. Gire and E. Price, Structural features of algebraic quantum notations, Phys. Rev. ST Phys. Educ. Res. 11, 020109 (2015).

[24] G. Passante, P. Emigh, and P. Shaffer, Examining student ideas about energy measurements on quantum states across undergraduate and graduate levels, Phys. Rev. ST Phys. Educ. Res. 11, 020111 (2015).

[25] E. Marshman and C. Singh, Framework for understanding the patterns of student difficulties in quantum mechanics, Phys. Rev. ST Phys. Educ. Res. 11, 020119 (2015).

[26] C. Singh and E. Marshman, Review of student difficulties in upper-level quantum mechanics, Phys. Rev. ST Phys. Educ. Res. 11, 020117 (2015).

[27] E. Marshman and C. Singh, Interactive tutorial to improve student understanding of single photon experiments involving a Mach-Zehnder interferometer, Eur. J. Phys. 37, 024001 (2016).

[28] E. Marshman and C. Singh, Investigating and improving student understanding of the probability distributions for measuring physical observables in quantum mechanics, Eur. J. Phys. 38, 025705 (2017).

[29] E. Marshman and C. Singh, Investigating and improving student understanding of the expectation values of observables in quantum mechanics, Eur. J. Phys. 38, 045701 (2017).

[30] V. Dini and D. Hammer, Case study of a successful learner's epistemological framings of quantum mechanics, Phys. Rev. Phys. Educ. Res. 13, 010124 (2017).

[31] C. Keebaugh, E. Marshman, and C. Singh, Investigating and addressing student difficulties with the corrections to the energies of the hydrogen atom for the strong and weak field Zeeman effect, Eur. J. Phys. 39, 045701 (2018).

[32] C. Keebaugh, E. Marshman, and C. Singh, Investigating and addressing student difficulties with a good basis for finding perturbative corrections in the context of degenerate perturbation theory, Eur. J. Phys. 39, 055701 (2018).

[33] C. Keebaugh, E. Marshman, and C. Singh, Improving student understanding of a system of identical particles with a fixed total energy, Am. J. Phys. 87, 583 (2019).

[34] C. Keebaugh, E. Marshman, and C. Singh, Improving student understanding of fine structure corrections to the energy spectrum of the hydrogen atom, Am. J. Phys. 87, 594 (2019).

[35] E. Marshman and C. Singh, Validation and administration of a conceptual survey on the formalism and postulates of quantum mechanics, Phys. Rev. Phys. Educ. Res. 15, 020128 (2019).

[36] C. Keebaugh, E. Marshman, and C. Singh, Improving student understanding of corrections to the energy spectrum of the hydrogen atom for the Zeeman effect, Phys. Rev. Phys. Educ. Res. 15, 010113 (2019).

[37] S. DeVore and C. Singh, Interactive learning tutorial on quantum key distribution, Phys. Rev. Phys. Educ. Res. 16, 010126 (2020). 
[38] C. Singh, Interactive learning tutorials on quantum mechanics, Am. J. Phys. 76, 400 (2008).

[39] B. R. Brown and C. Singh, Development and evaluation of a Quantum Interactive Learning Tutorial on Larmor precession of spin, PERC Proc. (2015), pp. 47-50, https://doi.org/10.1119/perc.2014.pr.008.

[40] E. Mazur, Peer Instruction: A User's Manual (Prentice Hall, Upper Saddle River, N.J., 1997).

[41] C. H. Crouch and E. Mazur, Peer Instruction: Ten years of experience and results, Am. J. Phys. 69, 970 (2001).

[42] P. Justice, E. Marshman, and C. Singh, Development and validation of a sequence of clicker questions for helping students learn addition of angular momentum in quantum mechanics, PERC Proc. (2018),

https://doi.org/10.1119/perc.2018.pr.Justice.

[43] P. Justice, E. Marshman, and C. Singh, Improving student understanding of quantum mechanics underlying the Stern-
Gerlach experiment using a research-validated multiplechoice question sequence, Eur. J. Phys. 40, 055702 (2019).

[44] P. Justice, E. Marshman, and C. Singh, Student understanding of Fermi energy, the Fermi-Dirac distribution and total electronic energy of a free electron gas, Eur. J. Phys. 41, 015704 (2019).

[45] P. Justice, E. Marshman, and C. Singh, Development, validation and in-class evaluation of a sequence of clicker questions on Larmor precession of spin in quantum mechanics, PERC Proc. (2019), pp. 269-274, https://doi.org/10.1119/perc.2014.pr.008.

[46] C. Singh and G. Zhu, Improving students' understanding of quantum mechanics by using peer instruction tools, AIP Conference Proceedings 1413, 77 (2012).

[47] J. Cohen, Statistical Power Analysis for the Behavioral Sciences (Academic Press, 2013). 